\title{
Quercetin inhibits intrahepatic cholangiocarcinoma by inducing ferroptosis and inhibiting epithelial- mesenchymal transformation via the NF-KB pathway
}

\section{Yinghui Song}

The First Affiliated Hospital of hunan normal University

Zhihua Zhang

The First People's Hospital of hunan normal university

\section{Qin Chai}

The First Affiliated Hospital of hunan normal University

\section{GuoYi Xia}

The First Affiliated Hospital of hunan normal University

\section{Zhangtao Yu}

The First Affiliated Hospital of hunan normal University

\section{Ranzhiqiang Yang}

The First Affiliated Hospital of hunan normal University

\section{Junkai Huang}

The First Affiliated Hospital of hunan normal University

\section{Yuhang Li}

The First Affiliated Hospital of hunan normal University

\section{Chuang Peng}

The First Affiliated Hospital of hunan normal University

\section{Bo Jiang}

The First Affiliated Hospital of hunan normal University

Sulai Liu ( $\nabla$ liusulai@hunnu.edu.cn )

Hunan Provincial People's Hospital/The First Affiliated Hospital of Hunan Normal University https://orcid.org/0000-0002-5257-3922

\section{Research}

Keywords: Intrahepatic cholangiocarcinoma, Quercetin, NF-kB, ferroptosis, EMT

Posted Date: October 5th, 2021 
DOI: https://doi.org/10.21203/rs.3.rs-947377/v1

License: (c) (1) This work is licensed under a Creative Commons Attribution 4.0 International License. Read Full License 


\section{Abstract}

Intrahepatic cholangiocarcinoma (ICC) is a rare high-fatal hepatobiliary malignancy, the treatment option of ICC is very limited, and the prognosis is also poor. Recently, emerging evidence has shown the potential of quercetin (QE) for cancer therapy. We explored the effect and mechanism of QE on ICC in vitro and in vivo. CCK-8 assay and Clonogenic assay showed that QE could inhibit ICC cells proliferation and survival. PI staining suggested QE could induce ICC cells arrest in G1 phase. AV/PI staining suggested QE could promote ICC cells apoptosis. Wound Healing Assay and Transwell chamber experiment suggested QE could inhibit ICC cells EMT. RNA-seq, the changes in the structure of mitochondria by electron microscopy and the key markers of ferroptosis (free iron ions, MDA, SOD, GPX4) were supported QE could promote ferroptosis in ICC cells. Molecular docking showed that QE had direct interaction with NF-KB and GPX4. In vivo, treatment with QE inhibited tumor growth and prolonged survival time of tumor-bearing nude mice. Our data for the first time suggest that QE is a new ferroptosis inducer and combinative treatment of inhibiting NF-KB in ICC cells by inducing ferroptosis and inhibiting EMT, which will hopefully provide a prospective strategy for ICC patients.

\section{Background}

Intrahepatic cholangiocarcinoma (ICC) is a primary tumor that originates from bile duct epithelial cells and ranks second in the incidence of primary liver tumors ${ }^{1}$. In recent years, the incidence of ICC has gradually increased especially in China ${ }^{2}$. Due to the insidious onset of ICC, it is mostly late disease when diagnosed, accompanied by nerve invasion and lymph node metastasis ${ }^{3}$. Moreover, the treatment options is very limited because ICC has low sensitivity to chemotherapy and radiotherapy ${ }^{4}$. At present, radical surgical resection is the most important method for the treatment of ICC ${ }^{5}$. However, the high recurrence rate after surgery leads to a five-year survival rate of less than $20 \%$ after surgery, and the overall ICC prognosis is extremely poor ${ }^{6}$. Therefore, it is important to explore the biological behavior of ICC and effective treatment methods to improve the therapeutic effect.

The risk factors of ICC including bile duct stones, hepatitis virus, bile duct cysts and liver cirrhosis, etc. ${ }^{7}$. It is well known that intrahepatic bile duct stones can induce ICC ${ }^{8}$. But the specific mechanism is still unclear. Accumulative evidences indicate that mechanical stimulation of stones and chronic inflammation of the bile duct caused by cholestasis can induce ICC ${ }^{9}$. The link between chronic inflammation and tumor is considered the seventh hallmark of malignancy. Chronic inflammation mediated by cytokines, chemokines, etc. leads to abnormal signal pathway activation, which is an important feature of tumor-related inflammation ${ }^{10}$. The transcription factor nuclear factor-KB (NF-KB) is an important mediator of innate immunity/inflammation. The dysregulation of the NF-kB pathway is related to many types of cancer ${ }^{11}$. NF-KB activation can induce the expression of inflammatory cytokines and adhesion molecules, together with the induction of NF-kB-mediated anti-apoptotic genes such as Bcl2 can promote tumor cell survival ${ }^{12}$. Also,it is confirmed that inhibiting NF-KB can play an anti-tumor effect in ICC ${ }^{13}$. 
Ferroptosis is a new type of programmed cell death that is iron-dependent and different from apoptosis, cell necrosis, and autophagy ${ }^{14}$. Ferroptosis is induced by peroxidative damage of cell membrane phospholipids causing by the accumulation of lipid reactive oxygen species in cells. It was reported and named by Dixon et al. in $2012^{15}$. The process of ferroptosis is accompanied by the accumulation of a large amount of iron and lipid peroxidation, which can be inhibited by iron chelating agents and antioxidants, but cannot be inhibited by apoptosis inhibitors ${ }^{16}$. Therefore, ferroptosis is an independent type of cell death that is iron-dependent and characterized by an abnormal increase in lipid reactive oxygen species ${ }^{17}$. The occurrence of ferroptosis involves lipid metabolism, the production and removal of reactive oxygen species, and abnormal iron metabolism. Inducing ferroptosis of cancer cells has gradually become a treatment strategy for many types of tumors ${ }^{18}$. However, the role of ferroptosis in ICC is temporarily unknown and needs further research.

Quercetin(QE) is a common flavonoid, which is widely present in plant flowers, stems, leaves, and fruits. It exists in the form of polyglycosides. The chemical formula of QE is $\mathrm{C}_{15} \mathrm{H}_{10} \mathrm{O}_{7}{ }^{19,20}$. QE plays an important role in relieving cough, relieving asthma, and reducing blood pressure, etc. ${ }^{21}$ Cumulative evidence have showed that QE could inhibit cancers including breast cancer and colon cancer by blocking the cell cycle, inducing tumor cell apoptosis, etc. ${ }^{22,23}$ However, the effect of QE on ICC is temporary unknown.

This study took ICC cells as the research object to observe the effect of QE on ICC, and explore the effect of QE on NF-KB and ferroptosis. This study provides a new experimental basis for in-depth understanding of the mechanism of QE in ICC.

\section{Materials And Method Cell Culture}

The intrahepatic cholangiocarcinoma cell lines HuCCT1, RBE and HCCC-9810 were obtained from the American Type Culture Collection (ATCC). ICC cells were cultured in 1640 medium containing $10 \%$ fetal bovine serum (FBS) and $100 \mathrm{U} / \mathrm{ml}$ penicillin/streptomycin. All the cells are grown in a $37^{\circ} \mathrm{C} / 5 \% \mathrm{CO} 2$ incubator.

\section{Reagents and Antibodies}

Quercetin (\#S2391), Ferrostatin-1(Fer-1) (\#S7243), RSL3 (\#S8155) were obtained from Selleck (Shanghai Lanmu Chemical Co., Ltd., Shanghai, China). CCK8 was purchased from Dojindo (Kumamoto, Japan, CK04). Annexin V/FITC apoptosis detection kit was purchased from Jiamay Biotech Co.LTD (Beijing, China, \#LHK601-050-P). Bay 11-7082 (\#S1523), lipopolysaccharide (LPS) (\#S1732), Lipid Peroxidation MDA Assay Kit (\#S0131S) and Total Superoxide Dismutase Assay Kit with WST-8 (\#S0101S) were purchased from Beyotime Biotechnology Company (Hangzhou, China). Iron assay kit was purchased from Abcam (ab83366). NF-kB (\#8242)/p-NF-kB (\#3033)/P21 (\#2947)/cleaved Caspase3 (\#9664)/E- 
cadherin (\#3195)/N-cadherin (\#13116)/GPX4 (\#52455)/ GAPDH(\#5174) were purchased from Cell Signaling Technology (Beverly, MA).

\section{CCK-8 assay for cell viability}

The CCK-8 reagent was used to determine the cell viability after QE treatment. ICC cells in exponential phase were seeded into 96 -well plates at a density of 5,000 cells per well. All the cells were divided into control group ( $0.1 \%$ mass fraction DMSO) or QE group (different concentrations), each group had 5 repeat wells. According to the manufacturer's instructions, CCK-8 was used to evaluate the cell proliferation activity after QE treatment. 24, 48, or 72 hours after QE treatment, $10 \mu \mathrm{L}$ CCK-8 reagent was added to each well, and the cells were incubated for another 1 hours. A microplate spectrophotometer (ELx800, BioTek Inc., USA) was used to measure the absorbance of the sample at $450 \mathrm{~nm}$, and each experiment was performed in triplicate.

\section{Clonogenic assay for cell survival rate}

The clone formation experiment was used to evaluate the effect of QE treatment on the proliferation ability of ICC clones. ICC cells were prepared as a single cell suspension and the cell density were counted. Next, $2 \mathrm{~mL}$ of culture medium was used to inoculate a 6-well plate at a density of 500 cells per well. After 24 hours, different concentrations of QE were added. After 24 hours of QE treatment, the drugcontaining mediums were removed, and the cells were cultured in the drug-free medium for 14 days. After fixing the cells with absolute ethanol, they were stained with Giemsa and counted manually. Colonies containing more than 50 cells were considered survivors.

\section{Cell cycle and apoptosis detection by flow cytometry}

The cell cycle was evaluated by using propidium iodide (PI) staining and flow cytometry (FCM) analysis. The ICC cells treated with different concentrations of QE for 24 hours were collected and fixed with $70 \%$ ethanol at $4^{\circ} \mathrm{C}$ for 24 hours. Next, the alcohol fixative solution was removed and the ICC cells were washed gently with cooled PBS twice. After that, the ICC cells were incubated with a dye solution containing $5 \mu \mathrm{g} / \mathrm{mL} \mathrm{PI}$ and $50 \mu \mathrm{g} / \mathrm{mL}$ RNase (Sigma Aldrich) for 30 minutes at room temperature in the dark. Then the cell cycle distribution was analyzed by using FACSuite analysis software (BD Bioscience) on BD Accuri C6. The experiments were repeated in triplicate.

Apoptosis was measured by Annexin V/FITC apoptosis detection kit according to the manufacturer's instruction. Briefly, ICC cells were treated with different concentrations of QE for 24 hours, then, cells were collected by $0.25 \%$ trypsin, washed with cold PBS twice, resuspended in $300 \mu \mathrm{L}$ Binding Buffer and stained with $5 \mu \mathrm{L}$ fluorescein isothiocyanate-labeled Annexin-V and $5 \mu \mathrm{L}$ PI. After 15 min of incubation, $200 \mu \mathrm{L}$ Binding Buffer was added before analyzed. Then the cell apoptosis ratio was analyzed by using FACSuite analysis software (BD Bioscience) on BD Accuri C6. The experiments were repeated in triplicate. qRT-PCR 
The qRT-PCR was performed as described in ${ }^{24}$. The cells were lysed, and total RNA was extracted using TRIzol reagent (Invitrogen, Carlsbad, CA, USA). Total RNA $(1 \mu \mathrm{g})$ was used for cDNA synthesis. RevertAid RT Reverse Transcription Kit (Thermo Fisher) was applied to perform RNA reverse transcription at $42^{\circ} \mathrm{C}$ for 6 minutes, and then qRT-PCR was performed on the resulting CDNA by using a SYBR-Green fluorescence-based Assay kit (Applied Biosystems, Foster City, CA, USA) and an ABI Prism 7500 Sequence Detection System (Applied Biosystems). The primer sequences were shown in the Supplementary Table 1. The amplification level of GAPDH was used as an endogenous control to normalize the amplification of the target gene. The relative quantification (RQ) of the target gene was calculated according to the threshold cycle (Ct) value as follows: $\mathrm{RQ}=2^{-\Delta \Delta \mathrm{Ct}}$, where $\Delta \Delta \mathrm{Ct}=$ [Ct (target gene)-Ct (GAPDH)] sample(treatment)-[Ct (target gene) -Ct(GAPDH)] sample (control).

\section{Western blotting}

Western blotting was performed as previously described ${ }^{25}$. The whole cell protein was extracted by using cell lysis buffer. The protein concentration was determined by using the method described in the Micro BCA protein determination kit (Thermo Fisher Scientific Inc, USA). Total protein $(20 \mu \mathrm{g})$ was loaded in each well. The following antibodies and diluents were used: anti-NF-KB $(1: 1,000)$, anti-p-NF-KB $(1: 1,000)$, antiP21 (1:1,000), anti-cleaved Caspase3 (1:1,000), anti-E-cadherin (1:1,000), anti-N-cadherin $(1: 1,000)$, antiGPX4 (1:1,000). Anti-GAPDH $(1: 2,000)$ as a loading control.

\section{Wound Healing Assay}

Cells were seeded into 6 -well plates at a density of $5^{\star} 10^{6}$ cells per well with $2 \mathrm{~mL}$ medium. After 24 hours, the medium was removed. And a micropipette tip was used to scratch the surface of the adherent ICC cells along the central axis of the 6-well plate. Next, the QE-containing 1640 medium was added to the 6well plate and continue culturing for 48 hours. Then the pictures were taken under a microscope. The Image $\mathrm{J}$ software was applied to analyze the scratch healing area, and the 48-hour cell scratch healing rate was calculated. Scratch healing rate/\%= (initial scratch width- $48 \mathrm{~h}$ scratch width )/Initial scratch width $\times 100 \%$. The experiments were repeated in triplicate.

\section{Transwell chamber experiment to detect the number of migrating cells}

Cells were seeded into 6-well plates at a density of $5 * 10^{6}$ cells per well with $2 \mathrm{~mL}$ medium. After 24 hours, QE-containing medium was replaced to the medium and the cells were cultured for another 24 hours. And the cells were digest and $2.5 \times 10^{4}$ cell suspension to the upper chamber of Transwell, $500 \mu \mathrm{L}$ complete medium containing $10 \%$ FBS was added to the lower chamber. The cells were cultured for another $48 \mathrm{~h}$. After that, the chamber was taken out and washed with PBS. Next, the cells were fixed with $4 \%$ paraformaldehyde for $15 \mathrm{~min}$, washed 3 times with PBS, stained with $0.1 \%$ crystal violet for $10 \mathrm{~min}$, washed 3 times with PBS. Then the pictures were selected randomly under a microscope. The number of migrated cells were counted. The experiments were repeated in triplicate.

\section{RNA-seq}


TRIzol (Invitrogen) method was used for total RNA extraction. After all the samples were qualified, the AMPure XP bead was used for purification, and finally PCR amplification was performed to obtain the final cDNA library. After the library was qualified, the different libraries were pooled according to the effective concentration and target offline data volume, and then sequenced on the HiSeq platform. The sequencing strategy was PE150. The clean reads were aligned to the reference genome using the software Hisat2, and the genome file was hg38. To analysis the distribution of reads on the genome, each chromosome was divided into $100 \mathrm{~kb}$ segments, and the number of reads located in the segment was statistically compared. The R package used for plotting is ggplot2. StringTie software was used to search the new transcripts, and the genome annotation version was hg38 gencode.v34. The Swiss-prot protein database was used to annotate the new transcripts with Diamond software, E value was $1 \mathrm{e}-5$. Gene expression calculation was performed by StringTie software. Gene expression profiling was based on the number of reads. TPM (fragments per kb of exon model per million mapped reads) values were used to estimate the expressed values and transcript levels. DESeq2 was selected to identifying differentially expressed genes (DEGs). Genes with an adjusted $P$ value $<0.05$ and abs (log2(fold change) $)>1$ were assigned as DEGs. Gene ontology (GO) terms are distributed into biological processes (BP), cellular components (CC) and molecular functions (MF). KEGG (Kyoto Encyclopedia of Genes and Genome) is the main public database related to the pathway. GO and KEGG functional enrichment analysis were performed on the cluster Profile R package. The ggplot2 and path view R package were select for plotting. The rMATs software was used to search alternative splicing event and calculate expression of each one. Alternative splicing event with $P$ value $<0.05$ and abs (Lnc Level Diffence) $>0.1$ were assigned as DE alternative splicing event. Picard - tools v1.41 and samtools v0.1.18 were used to sort, remove duplicated reads and merge the bam alignment results of each sample. GATK3.8 software was used to perform SNP calling. Raw vcf files were filtered with GATK standard filter method and other parameters (cluster Window Size: 10; MQ0 >= 4 and (MQ/(1.0*DP)) > 0.1; QUAL < 10; QUAL < 30.0 or QD < 5.0 or HRun > 5), and only SNPs with distance $>5$ were retained.

\section{Iron assay}

According to the manufacturer's instructions, the iron assay kit is used to determine the level of ferrous ions $\left(\mathrm{Fe} 2^{+}\right)$in cells. The cells were seeded on a six-well plate $\left(5 \times 10^{6}\right.$ cells per plate) and treated with DMSO, QE, Fer- 1 or RSL3 for 24 hours. The cells were collected and washed in cold PBS, homogenized in $5 x$ volume iron assay buffer on ice, and then centrifuged at $4^{\circ} \mathrm{C}(13,000 \mathrm{xg}, 10$ minutes) to remove insoluble materials. After collecting the supernatant, iron reducing agent was added to each sample, mixed and incubated at room temperature for 30 minutes. Add $100 \mu \mathrm{L}$ of iron probe to each sample. Use a horizontal shaker or pipette to mix thoroughly, and then incubate the reaction solution for 60 minutes at room temperature in the dark. Use a microplate reader to measure the absorbance at $593 \mathrm{~nm}$.

\section{Malondialdehyde(MDA) analysis}

The relative MDA concentration in cell lysates was assessed using a Lipid Peroxidation (MDA) Assay Kit according to the manufacturer's instructions. Briefly, MDA in the sample reacts with thiobarbituric acid 
(TBA) to generate a MDA-TBA adduct. The MDA-TBA adduct can be quantified colorimetrically (OD = 530 $\mathrm{nm})$.

\section{Superoxide Dismutase(SOD) Assay Kit with WST-8}

SOD Assay Kit-WST method was used for evaluation of SOD activity according to the manufacturer's instructions. The absorbance was assessed at $450 \mathrm{~nm}$ using a microplate reader.

\section{Transmission electron microscopy}

HuCCT1 was treated with $100 \mu \mathrm{mol} / \mathrm{L}$ QE for $24 \mathrm{~h}$ as previous method, and the cells were harvested for transmission electron microscopy analysis. Briefly, the cells were fixed in the electron microscope fixation solution (Google Bio, Wuhan, China, \#G1102) for $2-4$ hours at $4^{\circ} \mathrm{C}$. Then fixed with $1 \%$ osmic acid $\cdot 0.1 \mathrm{M}$ PBS (PH7.4) at room temperature $\left(20^{\circ} \mathrm{C}\right)$ for $2 \mathrm{~h}$. Next, the samples were sequentially dehydrated with 50\%-70\%-80\%-90\%-95\%-100\%-100\% alcohol-100\% acetone-100\% acetone for 15 minutes each time. After that, the samples were penetrated and embedded. Ultra-thin microtome (Leica, Leica UC7) is used to cut the sample into $60-80 \mathrm{~nm}$ slices. The slices were stained with uranium and lead ( $2 \%$ uranyl acetate saturated alcohol solution, lead citrate, each stained for 15 minutes), then dried overnight at room temperature. At last, the slices were observed under a transmission electron microscope (Hitachi, HT7700), and collected images for analysis. At least 10 images were acquired for each structure of interest and representative images are shown.

\section{Immunochemistry(IHC)}

For IHC, the tumor tissues were stored in $4 \%$ paraformaldehyde for over $24 \mathrm{~h}$ and cut into $4 \mathrm{~mm}$ thick slices. Paraffin wax was then removed and stained with IHC by a method described previously ${ }^{10}$.

\section{Molecular docking}

The crystal structure of NF-KB and GPX4 were retrieved from previous studies ${ }^{26,27}$. Ligands were removed from the protein by PyMol software and save the molecule as a PDB file. Proteins docking was analysed by AutoDock vina software and AutoDock Tools 1.5.6 software. Chim3D (2010) software was used to draw ligands (QE) and save ligands as a PDB file. The number of points and center grid box were shown in Table. Spacing was set to 1. Results were analyzed by PyMol software.

\section{Tumor xenograft assay}

HuCCT1 cells were subcutaneously injected into the upper flank of 4-5 weeks old nude mice (Shanghai Laboratory Animal Center; Shanghai, China). Then mice were divided into control group, QE $25 \mathrm{mg} / \mathrm{kg}$ group and QE $50 \mathrm{mg} / \mathrm{kg}$ group and 12 mice in each group ${ }^{28}$. The size of tumor xenograft was checked every 3 day. 5 of each group were sacrificed four weeks after seeding. The tumor xenografts were resected and weighed. Tumor volume was analyzed using the following formula: tumor volume $\left(\mathrm{mm}^{3}\right)=$ (length $\times$ width $\left.^{2}\right) / 2$. For survival analysis, the other 7 mice of each group were monitored daily, and the survival rate was recorded. All experimental procedures were approved by the Ethics Committee of Hunan Provincial People's Hospital/The First Affiliated Hospital of Hunan Normal University. 


\section{Statistical analysis}

All data shown represent the results of at least three independent experiments. The results were expressed as the mean plus or minus the standard deviation (SD). (SPSS) software was applied to analyze the statistical analysis of the data. One-way analysis of variance was used to check the statistical significance of the differences between the groups, and the p-value was equal to or less than 0.05 as statistically significant. Image $J$ was used to analyze the scratch healing area. All the diagrams were generated using GraphPad Prism 5 software (GraphPad Software, Inc., La Jolla, CA, USA).

\section{Results}

Quercetin (QE) inhibited the proliferation of ICC cells

After treating ICC cells with different concentrations of QE, the CCK8 assay found that QE had inhibitory effects on the proliferation of ICC cells in time- and dose-dependent manners (Figure 1A-C). HuCCT1 cells are the most sensitive to QE. The specific inhibitory concentration of QE was shown Figure D. Different concentrations of QE were administrated to add into the ICC cells, and the colony formation test found that QE could inhibit the colony formation of ICC cells (Figure 1E-G). Interestingly, the concentration of $100 \mu \mathrm{mol} / \mathrm{L}$ had a relatively limited effect on inhibiting proliferation, but its effect on inhibiting clone formation was very obvious, suggesting that the effect of QE on ICC seemed to have a continuous cumulative effect.

QE induced ICC cells to arrest in G1 phase and apoptosis

After treating ICC cells with different concentrations of QE for $24 \mathrm{~h}$, the cell cycle results showed that QE could induce more ICC cells to block in G1 phase (Figure 2A-C), and the apoptosis results indicated that QE could be induced ICC cells apoptosis (Figure 2E-G). Meanwhile, QE could up-regulate P21 and Caspase3 (Figure 2D,H). Similar to the trends of CCK8 and CFA, HuCCT1 was more sensitive to QE, including the effect of QE on cell cycle and apoptosis.

QE inhibited the invasion and metastasis of ICC cells

After treating ICC cells with different concentrations of $Q E$, the wound healing results showed that $Q E$ could inhibit the migration of ICC cells (Figure $3 \mathrm{~A}$ ). The results of Transwell showed that QE could inhibit the invasion and migration of ICC cells (Figure 3B). Considering the meaningful of EMT regulators, Ecadherin and $\mathrm{N}$-cadherin were analyzed by Western Blot. As we expect, QE could up-regulate E-cadherin, down-regulate $\mathrm{N}$-cadherin (Figure $3 \mathrm{C}$ ).

RNA-seq revealed the mechanism of QE on ICC

In order to further clarify the mechanism of QE inhibiting ICC, HuCCT1 cells were treated with $100 \mu \mathrm{mol} / \mathrm{L}$ QE and total RNA was extracted by TRIZOL method. RNA-seq technology was used to screen for differential mRNA in the control group (C) and QE group (QE). It was found that there were 56 up- 
regulated genes, 72 down-regulated genes, and 13,895 meaningless genes (see Figure 4A-D and Supplementary Table 2). It also listed top 5 genes that were significantly up-regulated or down-regulated after QE treatment (Figure 4E,G). Furthermore, qRT-PCR was applied to verify the top 5 differentially expressed (up-regulated and down-regulated) genes and found that: EID3, AKR1C2, AKR1B10, AKR1C1, CYP4F11 were significantly up-regulated in the QE treatment group, while TICAM2, OCLN, CDK3, PKP1 and KRT5 were significantly down-regulated in the QE treatment group (Figure 4F,H).

\section{QE inhibited ICC by inhibiting NF-KB}

NF-KB signaling pathway is a regulator of immune response and inflammation, and is closely related to the carcinogenic process of intrahepatic cholangiocarcinoma ${ }^{29}$. RNA-seq results indicated that TICAM2 was significantly down-regulated after QE treatment. Also, TICAM2 is convinced as a key upstream factor regulating NF-KB ${ }^{30}$. It is speculated that QE may play an anti-tumor effect by down-regulating the activity of NF-KB signaling pathway via TICAM2 regulation. We treated HuCCT1 cells with different concentrations QE, and observed the activity of NF-KB by Western Blot, and found that QE treatment could down-regulate $\mathrm{p}-\mathrm{NF}-\mathrm{KB}$ in a dose-dependent manner (Figure 5A). The molecular docking results show that QE could directly bind to NF-KB, the binding energy was $-7.6 \mathrm{kcal} / \mathrm{mol}$, and the specific binding sites were shown in Supplementary Table 3 (Figure 5B). Treating HuCCT1 cells with QE combined with NF-KB inhibitors could enhance the effects of QE in inducing $\mathrm{G} 1$ phase arrest, promoting apoptosis, and inhibiting invasion and metastasis, otherwise, treating HuCCT1 cells with QE combined with NF-KB inhibitors activator could partially neutralize the effects of QE in inducing $\mathrm{G} 1$ phase arrest, promoting apoptosis, and inhibiting invasion and metastasis (Figure 5D-G). Meanwhile, the effects were accompanied by changes in p21, cleaved-caspase3, E-cadherin and N-cadherin (Figure 5C).

QE promoted ferroptosis in ICC cells

RNA-seq results indicated that QE had a significant effect on the ferroptosis pathways of HuCCT1 cells (Figure 6A). We then detected the expression levels of GPX4 after treating QE, and found that QE downregulated the expression of GPX4 (Figure 6B). The molecular docking results show that QE could directly bind to GPX4, the binding energy was $-6.6 \mathrm{kcal} / \mathrm{mol}$, and the specific binding sites were shown in Supplementary Table 3 (Figure 6C). And we observed the HuCCT1 cells treated with QE by electron microscope. The results showed that the cells treated with QE showed obvious signs of ferroptosis, including large-scale damage and disintegration of cell membranes, and most of the mitochondria shrank and became smaller with membrane density. Increase, high electron density in the film, iron deposition, and ridge expansion (Figure 6D). Meanwhile, QE treatment could increase intracellular iron ions, MDA levels and decrease SOD level (Figure 6E-G). Moreover, treatment of HuCCT1 cells with QE combined with ferroptosis promoters (RSL3) could increase ferroptosis promoted by QE, and ferroptosis scavengers (Fer-1) could partially neutralize ferroptosis promoted by QE, indicating that QE participated in promoting ferroptosis in ICC cells (Figure $6 \mathrm{H}-\mathrm{J}$ ). Meanwhile, the effects were accompanied by changes in GPX4 (Figure 6K). Moreover, regulating NF-KB had a regulatory effect on GPX4, considering the key effect on ferroptosis, suggesting that QE may promote ferroptosis by inhibiting NF-KB (Figure 6L). 
Subcutaneous tumors in nude mice were constructed, and the mice were treated with different concentrations of QE after tumor formation. It was found that QE could inhibit tumor size without affecting the weight of mice, indicating that QE has anti-tumor activity in vivo (Figure 7A-C). According to the results of the colony formation assay, QE seemed had a continuous effect. We observed the survival time of tumor-bearing nude mice and found that QE could significantly prolong the survival time of mice (Figure 7D). Also, the IHC results showed QE could down-regulate P-NF-KB and GPX4, which consistence with results in vitro (Figure 7E).

\section{Discussion}

Intrahepatic cholangiocarcinoma (ICC), a rare high-fatal hepatobiliary malignancy, has become the focus of attention in recent years because of its rising incidence and high mortality in the worldwide ${ }^{2,3}$. However, the treatment option of cholangiocarcinoma is very limited, and the prognosis is also poor ${ }^{31}$. This research revealed that QE could significantly inhibit the proliferation of ICC, induce the block of G1 phase, induce cell apoptosis and inhibit EMT and promote ferroptosis, It provides a new perspective for the treatment of cholangiocarcinoma.

QE has been shown to have anti-inflammation effects in a variety of disease models ${ }^{32}$. Recently, QE has been confirmed to have anti-tumor activity in a variety of tumors ${ }^{33,34}$. In fact, as early as 1999 , it is reported that QE could inhibit the proliferation of HuCCT $1^{35}$. However, the specific mechanism is temporarily undefined.

Through RNA-seq, it was found that TICAM2 was down-regulated particularly in HuCCT1 cells treated with QE. Meanwhile,TICAM2 is convinced as a key upstream factor regulating NF-KB ${ }^{30}$. Considering the role of NF-KB in inflammation and the role of chronic inflammation in the pathogenesis of cholangiocarcinoma, we investigated the effect of QE on NF-KB. As we expected, QE can significantly down-regulate the expression of phosphorylated NF-KB and regulate the key molecules downstream of the NF-KB signaling pathway including P21, Caspase3, E-cadherin, and N-cadherin ${ }^{36-39}$, which is contribution to QE play a role in inhibiting ICC.

Ferroptosis, a novel form of regulating cell death, is the connection among metabolism, redox biology and human diseases ${ }^{40}$. New evidence illustrated that it might lead to ferroptosis in cancer-treatment, especially in treating malignant tumors that are resistant to traditional therapies ${ }^{41}$. Ferroptosis-inducing factors could influence glutathione peroxidase by various pathways indirectly or directly, leading to a decrease in antioxidant capacity and accumulation of lipid reactive oxygen species (ROS) in cells, finally resulting in oxidative cell death ${ }^{42}$. Thence, ferroptosis plays a vital role in ICC treatment. QE induces EBmediated lysosomal activation to increase degradation of ferritin, which results in ferroptosis ${ }^{43}$. Meanwhile, QE can be transformed into a ferritin inhibitor through the antioxidant pathway to result in ferroptosis ${ }^{44}$. 
BAY11-7082 inhibits the phosphorylation of IKB-a, which is essential for the release of NF-KB from the cytosolic 1 KB- $\mathrm{a} / \mathrm{NF}-\mathrm{kB}$ complex ${ }^{45}$. Our results showed that inhibition NF-KB by BAY11-7082 could induce ferroptosis in HuCCT1 cells. Moreover, activation of NF-KB by LPS could inhibit ferroptosis, which was consistent with the situation of septic shock ${ }^{46}$. The inhibition of GPX4 activity could lead the subsequent activation of ferroptosis ${ }^{47}$. In our study, QE could inhibit GPX4 directly and inaction of NF-KB indirectly. Moreover, molecular docking results showed that QE could directly bind to GPX4, indicating that QE could inhibit GPX4 directly. Nonetheless, QE promote ferroptosis in through multiple mechanisms, and more indepth exploration is needed.

\section{Conclusion}

In summary, our data for the first time suggest that QE is a new ferroptosis inducer and combinative treatment of inhibiting NF-KB in ICC cells by inducing ferroptosis and inhibiting EMT, which will hopefully provide a prospective strategy for ICC patients (figure 7F).

\section{Abbreviations}

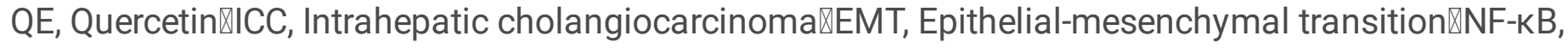

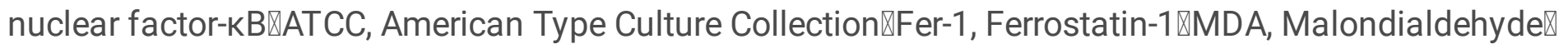
LPS, lipopolysaccharide $\triangle P I$, propidium iodide $\mathbb{F C M}$, flow cytometry $\mathbb{R Q}$, quantification $\triangle F B S$, Fetal Bovine Serum; DEG, differentially expressed gene; GO, Gene ontology; KEGG, Kyoto Encyclopedia of Genes and Genome; TBA, thiobarbituric acid; SOD, Superoxide Dismutase; SD, standard deviation; IHC, immunochemistry

\section{Declarations}

\section{Ethics approval and consent to participate}

All procedures performed in studies involving human participants were in accordance with the ethical standards of the institutional and/or national research committee and with the 1964 Helsinki declaration and its later amendments or comparable ethical standards. This study was approved by the ethical committee of Hunan Normal University.

\section{Consent for publication}

Written informed consent for publication was obtained from all participants.

\section{Availability of data and materials}

Data and materials are included in the manuscript.

\section{Competing interests}


The authors have declared that no competing interest exists.

\section{Funding}

This work was financially supported by following funds: Key project of Hunan Administration of Traditional Chinese Medicine (201809); Youth Talent of Hunan (2020RC3066) ; Postdoctoral Innovation Talents Project (2020RC2064)凶Hunan Provincial Natural Science Foundation of China (2020JJ5610); Hunan Natural Science Fund for Excellent Young Scholars (2021JJ20003) ;China Postdoctoral Science Foundation (2020M68115/2021T140197);The Project of Improving the Diagnosis and Treatment Capacity of Hepatobiliary, Pancreas and Intestine in Hunan Province (Xiangwei [2019] No. 118);

\section{Authors' contributions}

Yinghui Song, Zhihua Zhang, Qin Chai and GuoYi Xia performed the experiment; Yinghui Song, Ranzhiqiang Yang and Zhangtao Yu contributed significantly to analysis and manuscript preparation; Junkai Huang and Yuhang Li performed the data analyses and wrote the manuscript; Chuang Peng, Bo Jiang and Sulai Liu contributed to the conception of the study; Yinghui Song and Sulai Liu helped perform the analysis with constructive discussions.

\section{Acknowledgements}

None

\section{References}

1. Lee $Y$, Wang J, Luu M, et al. Comparison of Clinical Features and Outcomes between Intrahepatic Cholangiocarcinoma and Hepatocellular Carcinoma in the United States. Hepatology (Baltimore, Md) 2021.

2. Zhu H, Ji K, Wu W, et al. Describing Treatment Patterns for Elderly Patients with Intrahepatic Cholangiocarcinoma and Predicting Prognosis by a Validated Model: A Population-Based Study. J Cancer. 2021;12(11):3114-25.

3. Jolissaint J, Soares K, Seier K, et al. Intrahepatic Cholangiocarcinoma with Lymph Node Metastasis: Treatment-Related Outcomes and the Role of Tumor Genomics in Patient Selection. Clinical cancer research: an official journal of the American Association for Cancer Research. 2021;27(14):4101-8.

4. Edeline J, Du F, Rayar M, et al. Glass Microspheres 90Y Selective Internal Radiation Therapy and Chemotherapy as First-Line Treatment of Intrahepatic Cholangiocarcinoma. Clinical nuclear medicine. 2015;40(11):851-5.

5. Liu S, Liu X, Li X, et al. Application of Laparoscopic Radical Resection for Type III and IV Hilar Cholangiocarcinoma Treatment. Gastroenterology research and practice 2020; 2020: 1506275. 
6. Ke Q, Lin N, Deng M, Wang L, Zeng Y, Liu J. The effect of adjuvant therapy for patients with intrahepatic cholangiocarcinoma after surgical resection: A systematic review and meta-analysis. PloS one. 2020;15(2):e0229292.

7. Zhang $\mathrm{H}$, Yang T, Wu M, Shen F. Intrahepatic cholangiocarcinoma: Epidemiology, risk factors, diagnosis and surgical management. Cancer letters. 2016;379(2):198-205.

8. Chen M, Jan Y, Wang C, Jeng L, Hwang T, Chen S. Intrahepatic stones associated with cholangiocarcinoma. Am J Gastroenterol. 1989;84(4):391-5.

9. Chung T, Rhee H, Nahm J, et al. Clinicopathological characteristics of intrahepatic cholangiocarcinoma according to gross morphologic type: cholangiolocellular differentiation traits and inflammation- and proliferation-phenotypes. HPB: the official journal of the International Hepato Pancreato Biliary Association. 2020;22(6):864-73.

10. Liu S, Jiang J, Huang L, et al. iNOS is associated with tumorigenicity as an independent prognosticator in human intrahepatic cholangiocarcinoma. Cancer management research. 2019;11:8005-22.

11. Karin M. Nuclear factor-kappaB in cancer development and progression. Nature. 2006;441(7092):431-6.

12. Lin W, Karin M. A cytokine-mediated link between innate immunity, inflammation, and cancer. J Clin Investig. 2007;117(5):1175-83.

13. Yu G, Wang X, Zheng S, et al. RA190, a Proteasome Subunit ADRM1 Inhibitor, Suppresses Intrahepatic Cholangiocarcinoma by Inducing NF-KB-Mediated Cell Apoptosis. Cellular physiology biochemistry: international journal of experimental cellular physiology biochemistry pharmacology. 2018;47(3):1152-66.

14. Niu B, Liao K, Zhou Y, et al. Application of glutathione depletion in cancer therapy: Enhanced ROSbased therapy, ferroptosis, and chemotherapy. Biomaterials. 2021;277:121110.

15. Dixon S, Lemberg K, Lamprecht M, et al. Ferroptosis: an iron-dependent form of nonapoptotic cell death. Cell. 2012;149(5):1060-72.

16. Yagoda N, von Rechenberg M, Zaganjor E, et al. RAS-RAF-MEK-dependent oxidative cell death involving voltage-dependent anion channels. Nature. 2007;447(7146):864-8.

17. Li P, Jiang M, Li K, et al. Glutathione peroxidase 4-regulated neutrophil ferroptosis induces systemic autoimmunity. Nature immunology 2021.

18. Lei G, Zhang Y, Koppula $P$, et al. The role of ferroptosis in ionizing radiation-induced cell death and tumor suppression. Cell research. 2020;30(2):146-62.

19. Yi H, Peng H, Wu X, et al. The Therapeutic Effects and Mechanisms of Quercetin on Metabolic Diseases: Pharmacological Data and Clinical Evidence. Oxidative medicine and cellular longevity 2021; 2021: 6678662.

20. Brovarets' O, Hovorun D. Intramolecular tautomerization of the quercetin molecule due to the proton transfer: QM computational study. PloS one. 2019;14(11):e0224762. 
21. Zhou D, Luo M, Shang A, et al. Antioxidant Food Components for the Prevention and Treatment of Cardiovascular Diseases: Effects, Mechanisms, and Clinical Studies. Oxidative medicine and cellular longevity 2021; 2021: 6627355.

22. Mohammed H, Sulaiman G, Anwar S, et al. Quercetin against MCF7 and CAL51 breast cancer cell lines: apoptosis, gene expression, and cytotoxicity of nano-quercetin. Nanomedicine (London, England) 2021.

23. Shree A, Islam J, Sultana S. Quercetin ameliorates reactive oxygen species generation, inflammation, mucus depletion, goblet disintegration, and tumor multiplicity in colon cancer: Probable role of adenomatous polyposis coli, $\beta$-catenin. Phytother Res. 2021;35(4):2171-84.

24. Song Y, Chai Q, Wang N, Yang F, Wang G, Hu J. X-rays induced IL-8 production in lung cancer cells via p38/MAPK and NF-KB pathway. Int J Radiat Biol. 2020;96(11):1374-81.

25. Song $Y$, Zhong $M, G a n$, et al. ALDH1A1 mediates resistance of diffuse large B cell lymphoma to the $\mathrm{CHOP}$ regimen. Tumour biology: the journal of the International Society for Oncodevelopmental Biology Medicine. 2014;35(12):11809-17.

26. Huxford T, Huang D, Malek S, Ghosh G. The crystal structure of the IkappaBalpha/NF-kappaB complex reveals mechanisms of NF-kappaB inactivation. Cell. 1998;95(6):759-70.

27. Moosmayer D, Hilpmann A, Hoffmann J, et al. Crystal structures of the selenoprotein glutathione peroxidase 4 in its apo form and in complex with the covalently bound inhibitor ML162. Acta crystallographica Section D Structural biology. 2021;77:237-48.

28. Zhang X, Huang J, Yu C, et al. Quercetin Enhanced Paclitaxel Therapeutic Effects Towards PC-3 Prostate Cancer Through ER Stress Induction and ROS Production. OncoTargets therapy. 2020;13:513-23.

29. Saengboonmee C, Phoomak C, Supabphol S, et al. NF-kB and STAT3 co-operation enhances high glucose induced aggressiveness of cholangiocarcinoma cells. Life sciences. 2020;262:118548.

30. Slattery M, Mullany L, Sakoda L, et al. The NF-KB signalling pathway in colorectal cancer: associations between dysregulated gene and miRNA expression. $J$ Cancer Res Clin Oncol. 2018;144(2):269-83.

31. Liu S, Jiang B, Li H, et al. Wip1 is associated with tumorigenity and metastasis through MMP-2 in human intrahepatic cholangiocarcinoma. Oncotarget. 2017;8(34):56672-83.

32. Wang Y, Li C, Wan Y, et al. Quercetin-Loaded Ceria Nanocomposite Potentiate Dual-Directional Immunoregulation via Macrophage Polarization against Periodontal Inflammation. Small (Weinheim an der Bergstrasse, Germany) 2021: e2101505.

33. Liu M, Fu M, Yang X, et al. Paclitaxel and quercetin co-loaded functional mesoporous silica nanoparticles overcoming multidrug resistance in breast cancer. Colloids surfaces B Biointerfaces. 2020;196:111284.

34. Wang Z, Ma J, Li X, et al. Quercetin induces p53-independent cancer cell death through lysosome activation by the transcription factor EB and Reactive Oxygen Species-dependent ferroptosis. $\mathrm{Br} \mathrm{J}$ Pharmacol. 2021;178(5):1133-48. 
35. Kudo M, Naito Z, Yokoyama M, Asano G. Effects of quercetin and sunphenon on responses of cancer cells to heat shock damage. Exp Mol Pathol. 1999;66(1):66-75.

36. Wei X, Luo D, Yan Y, et al. Kojic acid inhibits senescence of human corneal endothelial cells via NF-KB and p21 signaling pathways. Exp Eye Res. 2019;180:174-83.

37. Sangaran P, Ibrahim Z, Chik Z, Mohamed Z, Ahmadiani A. LPS Preconditioning Attenuates Apoptosis Mechanism by Inhibiting NF-KB and Caspase-3 Activity: TLR4 Pre-activation in the Signaling Pathway of LPS-Induced Neuroprotection. Mol Neurobiol. 2021;58(5):2407-22.

38. Menezes S, Fouani L, Huang M, et al. The metastasis suppressor, NDRG1, attenuates oncogenic TGF$\beta$ and NF-KB signaling to enhance membrane E-cadherin expression in pancreatic cancer cells. Carcinogenesis. 2019;40(6):805-18.

39. Hou G, Zhao H, Teng H, et al. N-Cadherin Attenuates High Glucose-Induced Nucleus Pulposus Cell Senescence Through Regulation of the ROS/NF-KB Pathway. Cellular physiology biochemistry: international journal of experimental cellular physiology biochemistry pharmacology. 2018;47(1):257-65.

40. Stoyanovsky D, Tyurina Y, Shrivastava I, et al. Iron catalysis of lipid peroxidation in ferroptosis: Regulated enzymatic or random free radical reaction? Free Radic Biol Med. 2019;133:153-61.

41. Labib P, Goodchild G, Pereira S. Molecular Pathogenesis of Cholangiocarcinoma. BMC Cancer. 2019;19(1):185.

42. Xu G, Wang H, Li X, Huang R, Luo L. Recent progress on targeting ferroptosis for cancer therapy. Biochem Pharmacol. 2021;190:114584.

43. Li J, Cao F, Yin H, et al. Ferroptosis: past, present and future. Cell death disease. 2020;11(2):88.

44. Li X, Zeng J, Liu Y, et al. antilnhibitory Effect and Mechanism of Action of Quercetin and Quercetin Diels-Alder -Dimer on Erastin-Induced Ferroptosis in Bone Marrow-Derived Mesenchymal Stem Cells. Antioxidants (Basel, Switzerland) 2020; 9(3).

45. Zhang Q, Mao Z, Sun J. NF-kB inhibitor, BAY11-7082, suppresses M2 tumor-associated macrophage induced EMT potential via miR-30a/NF-KB/Snail signaling in bladder cancer cells. Gene. 2019;710:91-7.

46. Oh B, Lee S, Park G, et al. Erastin Inhibits Septic Shock and Inflammatory Gene Expression via Suppression of the NF-KB Pathway. Journal of clinical medicine 2019; 8(12).

47. Kim D, Kim W, Kim S, Moon D, Lee S. TGF- 11 -mediated repression of SLC7A11 drives vulnerability to GPX4 inhibition in hepatocellular carcinoma cells. Cell death disease. 2020;11(5):406.

\section{Figures}


(A)

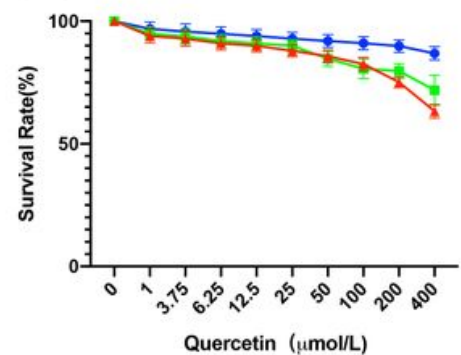

(D)

\begin{tabular}{|c|c|c|c|c|c|c|c|c|c|}
\hline & \multicolumn{3}{|c|}{$\mathrm{IC} 10(\mu \mathrm{M})$} & \multicolumn{3}{|c|}{$\mathrm{IC} 20(\mu \mathrm{M})$} & \multicolumn{3}{|c|}{ IC50 $(\mu \mathrm{M})$} \\
\hline & RBE & HCCC9810 & HuCCT1 & RBE & HCCC9810 & НuCCT1 & RBE & HCCC9810 & HuCCT1 \\
\hline $24 \mathrm{H}$ & 299.59 & 243.76 & 162.43 & 548.11 & 486.42 & 334.67 & 1023.55 & 952.76 & 664.52 \\
\hline $48 \mathrm{H}$ & 108.26 & 80.59 & 59.87 & 309.25 & 278.55 & 215.53 & 693.78 & 656.64 & 515.23 \\
\hline $72 \mathrm{H}$ & 51.22 & 35.88 & 28.45 & 182.67 & 166.73 & 141.79 & 434.15 & 417.05 & 358.77 \\
\hline
\end{tabular}

(E)
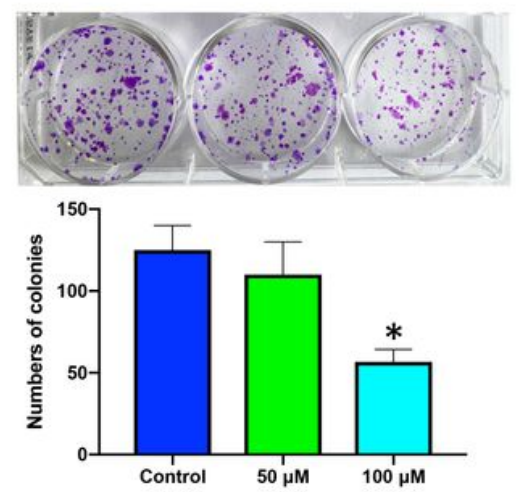

(B)

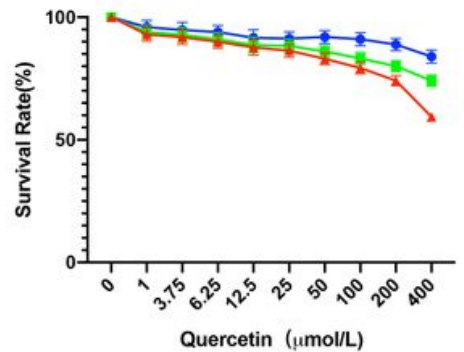

(C)

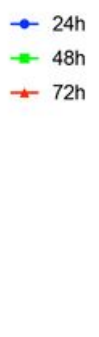

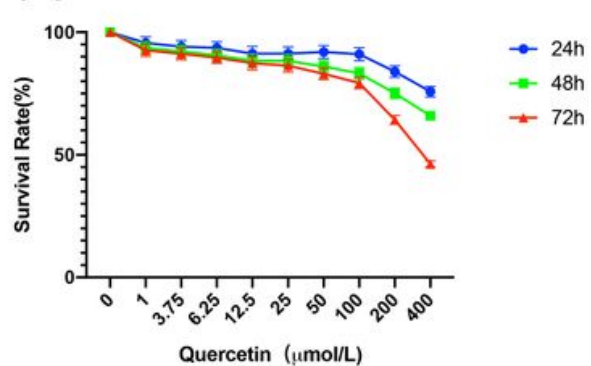

(F)
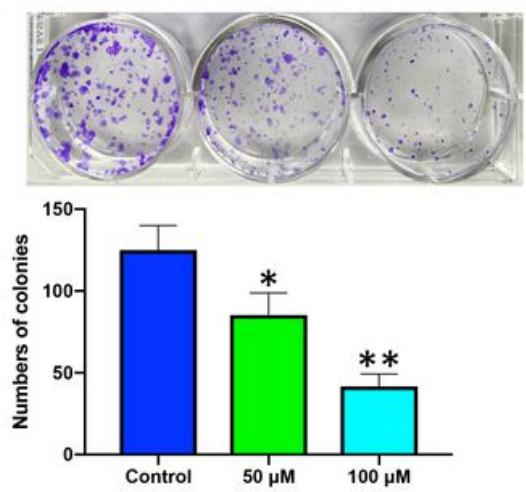

(G)
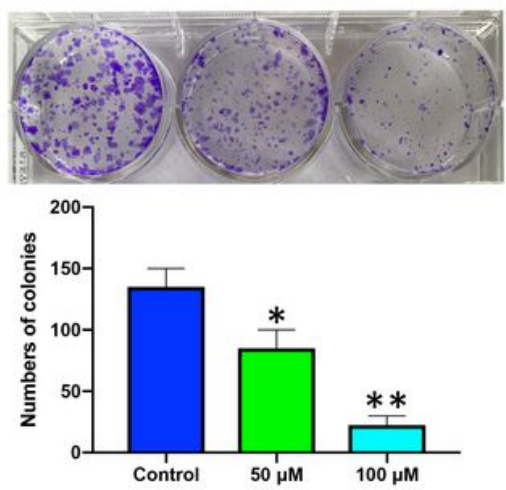

Figure 1

Quercetin (QE) inhibited the proliferation of ICC cells. (A-C) CCK8 assay was applied to analyzed QE on the proliferation of ICC cells (A.RBE cells, B.HCCC9810 cells, C.HuCCT1 cells). (D) The specific inhibitory concentration of QE. (E-G)The colony formation assay was used to analyzed QE on the colony formation of ICC cells (E.RBE cells, F.HCCC9810 cells, G.HuCCT1 cells). * Pख0.05, **Pख0.01. 
(A)

(B)

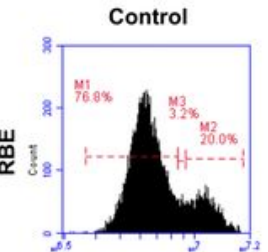

Control

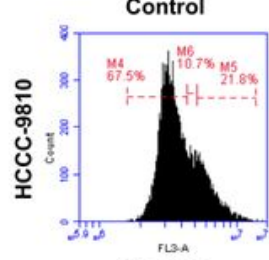

Control

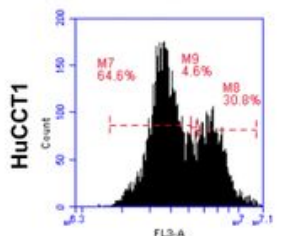

(D)

(E)
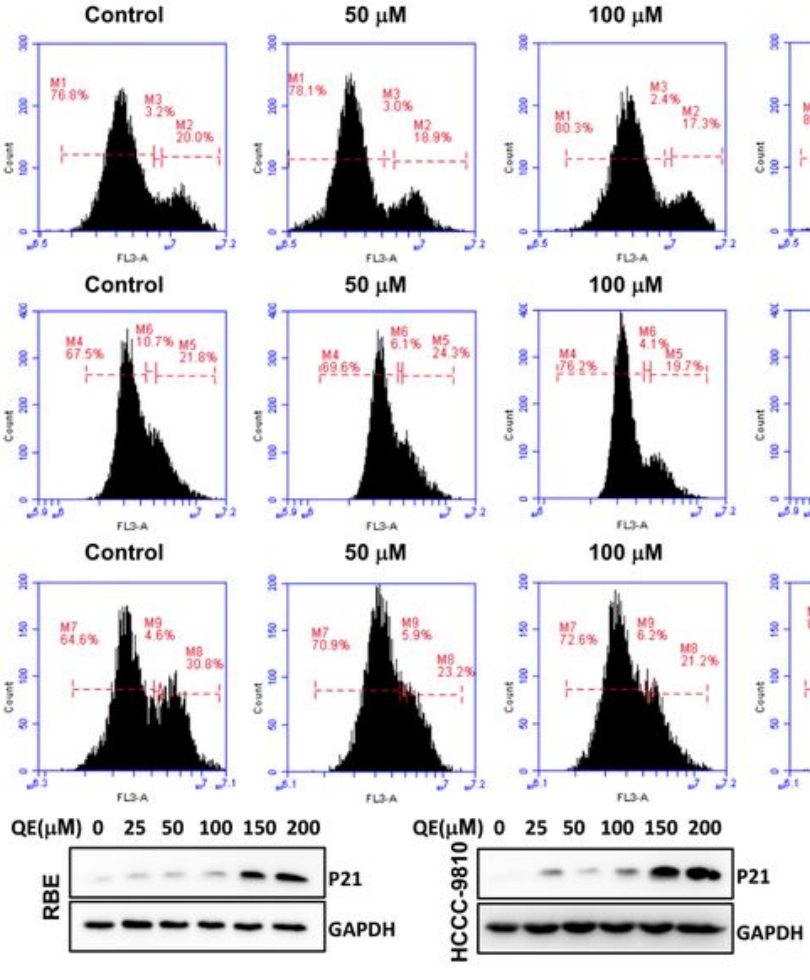

$50 \mu \mathrm{M}$
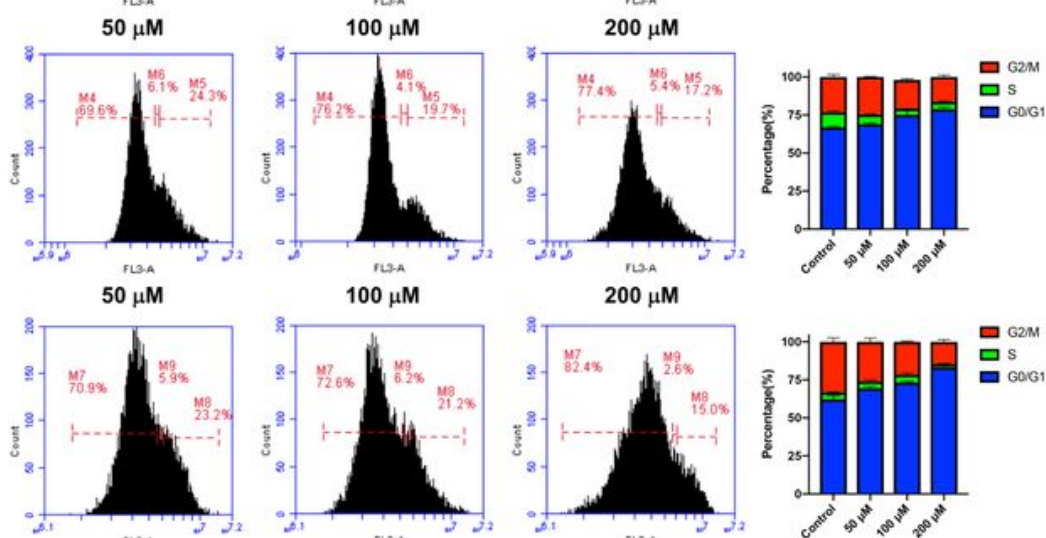

$200 \mu \mathrm{M}$

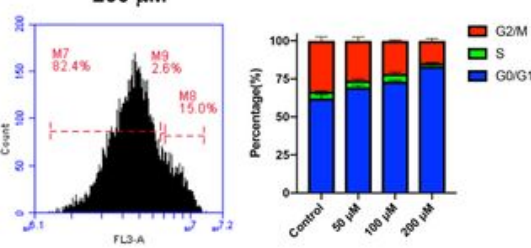

$\mathrm{QE}(\mu \mathrm{M}) 0 \quad 2550100150200$
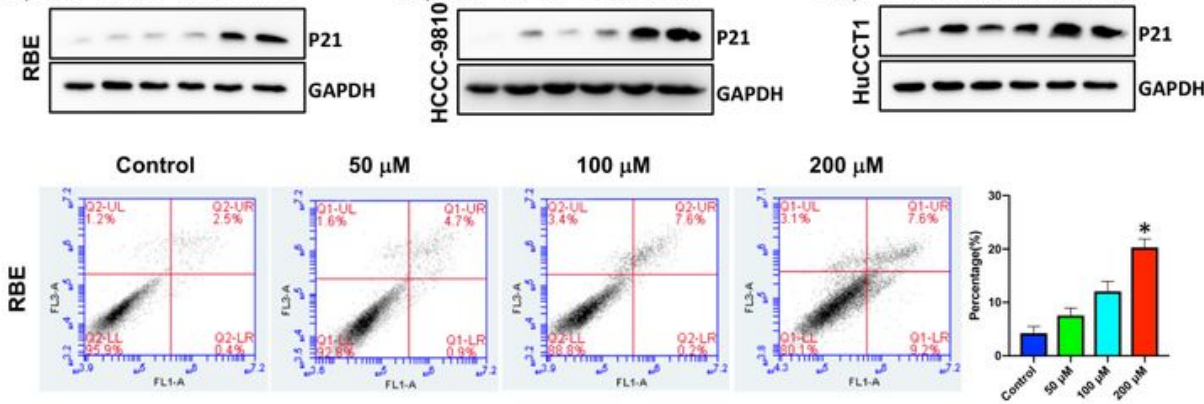

(F)
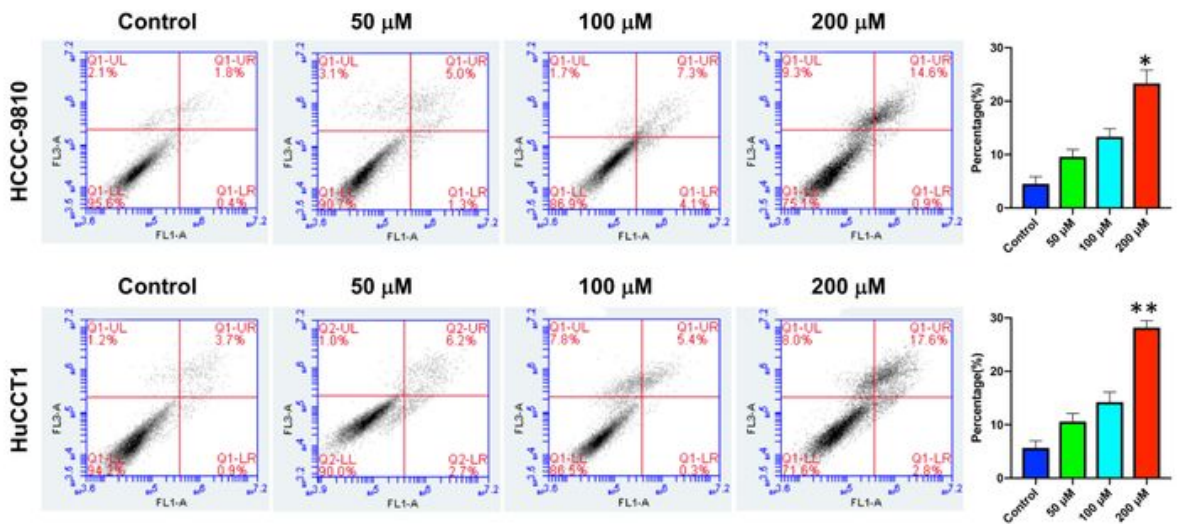

(H) $\quad \mathrm{QE}(\mu \mathrm{M}) 0 \quad 2550100150200$
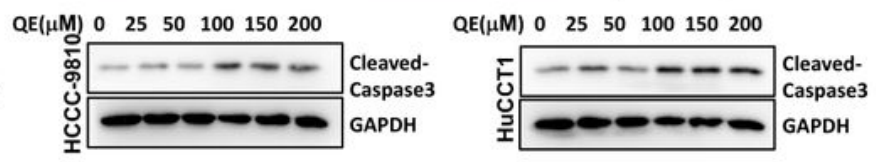

\section{Figure 2}

QE induced ICC cells to arrest in G1 phase and apoptosis. (A-C) PI staining was applied to analyze QE on the cell cycle distribution of ICC cells, and showed that QE could induce more ICC cells to block in $\mathrm{G} 1$ phase (A.RBE cells, B.HCCC9810 cells, C.HuCCT1 cells). (D) P21 was up-regulated by QE treatment.. (EG)Annexin V/FITC and PI staining was applied to analyzed QE on the cell apoptosis of ICC cells, and 
showed that QE could be induced ICC cells apoptosis (E.RBE cells, F.HCCC9810 cells, G.HuCCT1 cells. (H) Cleaved-caspase3 was up-regulated by QE treatment. * P®0.05, **P®0.01.

(A)
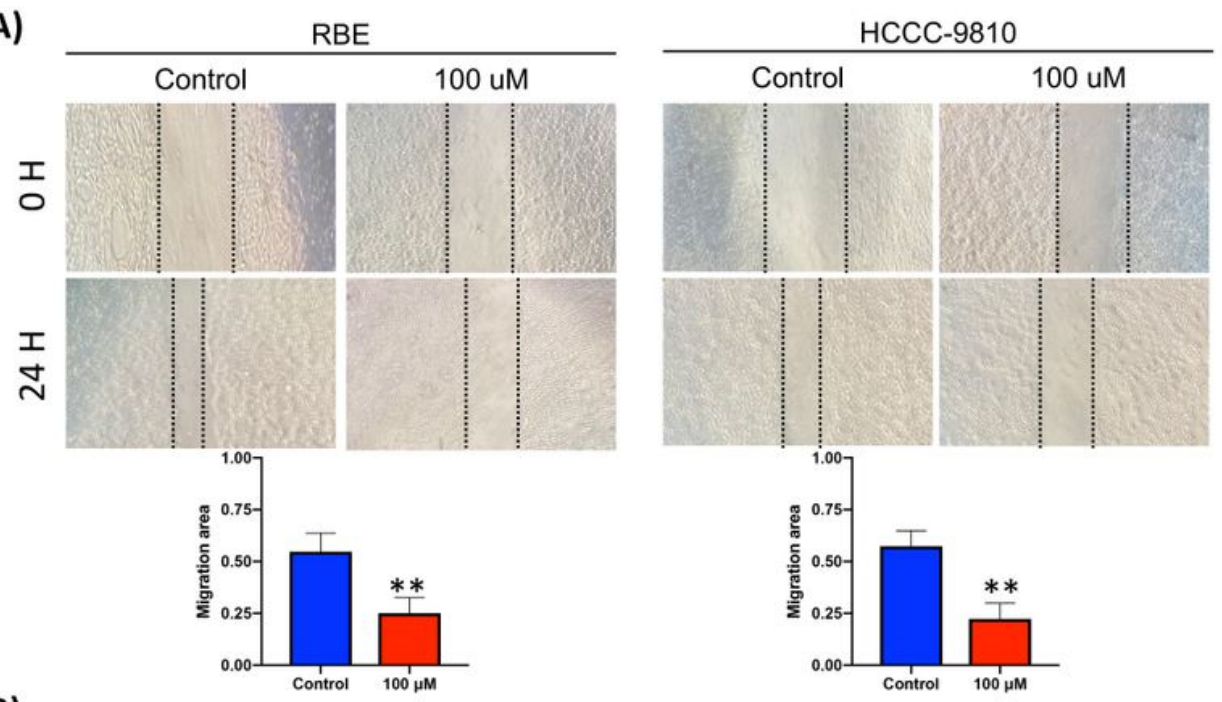

(B)
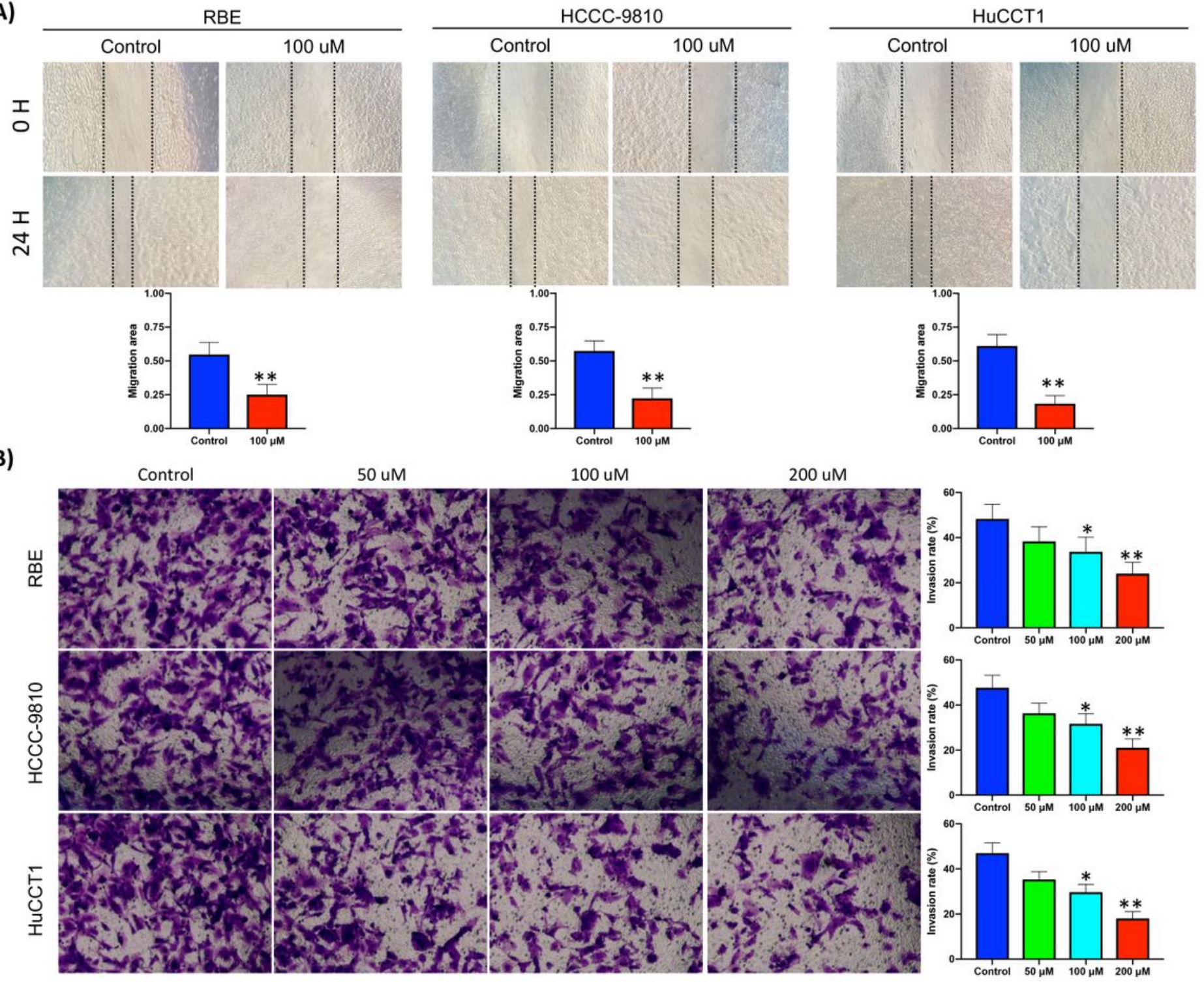

(C)
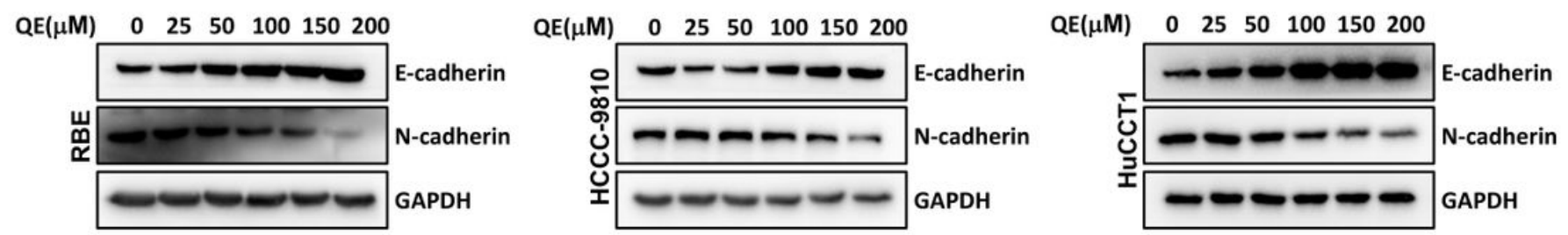

\section{Figure 3}

QE inhibited the invasion and metastasis of ICC cells. (A) Wound healing was used to analyze QE on the migration of ICC cells, and showed that QE could inhibit the migration of ICC cells. (B)Transwell was used to analyze QE on the invasion of ICC cells, and showed that QE could inhibit the invasion and migration of ICC cells. (C) E-cadherin was up-regulated by QE treatment and N-cadherin was down-regulated by QE treatment. * $\mathrm{P} \otimes 0.05$, **Pष0.01. 
(A)

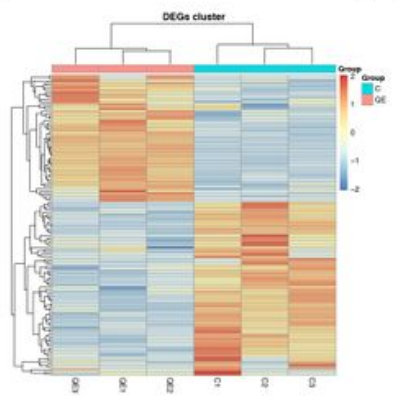

(B)

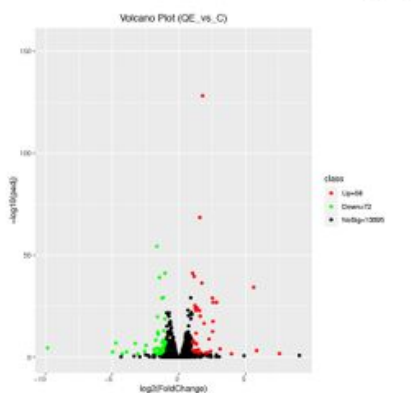

(C)

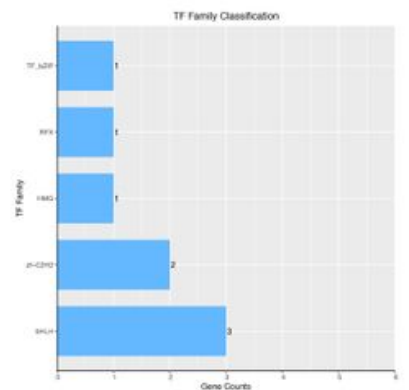

(D)

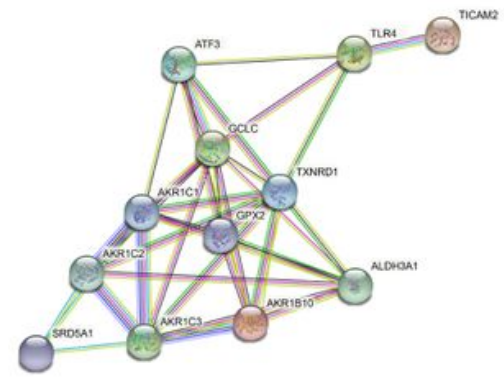

(E)

\begin{tabular}{lllll}
\hline Gene name & Control group & Quercetin group & Log2 (Ratio) & Function \\
\hline EID3 & 6.65 & 55.38 & 3.073 & Protein families: genetic information processing \\
AKR1C2 & 40.06 & 281.44 & 2.819 & Lipid metabolism \\
AKR1B10 & 207.92 & 1216.45 & 2.544 & Carbohydrate metabolism, Lipid metabolism \\
AKR1C1 & 63.29 & 359.41 & 2.516 & Lipid metabolism, Xenobiotics biodegradation and metabolism \\
CYP4F11 & 64.26 & 362.98 & 2.504 & Protein families: metabolism \\
\hline
\end{tabular}

(F)

(G)
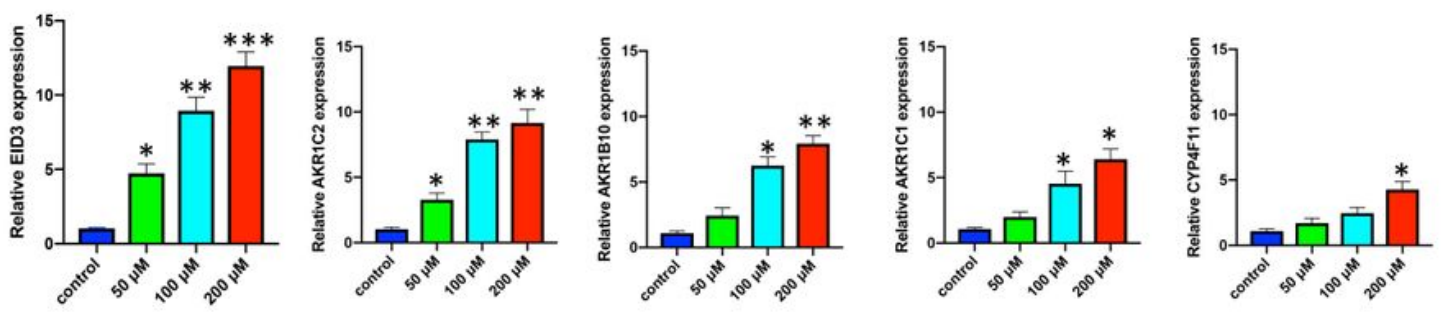

\begin{tabular}{lllll}
\hline Gene name & Control group & Quercetin group & Log2(Ratio) & \multicolumn{1}{l}{ Function } \\
\hline TICAM2 & 589.07 & 18.69 & -4.979 & $\begin{array}{l}\text { Signal transduction, Cell growth and death } \\
\text { OCLN }\end{array}$ \\
& 105.21 & 10.79 & -3.283 & $\begin{array}{l}\text { Protein families: signaling and cellular processes, } \\
\text { Signaling molecules and interaction }\end{array}$ \\
CDK3 & 97.26 & 26.33 & -1.885 & $\begin{array}{l}\text { Protein families: metabolism } \\
\text { PKP1 }\end{array}$ \\
KRT5 & 879 & 90.15 & -1.631 & Protein families: signaling and cellular processes \\
\hline
\end{tabular}

(H)
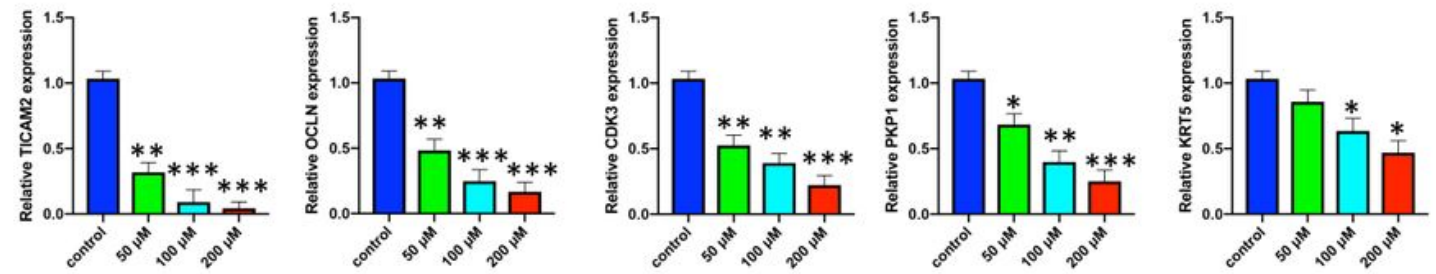

\section{Figure 4}

RNA-seq revealed the mechanism of QE on ICC. (A) the heatmap of differential mRNA in the control group (C) and QE group (QE). (B) the volcano map of differential mRNA in the C group and QE group. (C) the TF family classification. (D) the PPI. (E) the top 5 up-regulation gene. (F) q-RT-PCR for the top 5 up-regulation gene. $(\mathrm{G})$ the top 5 down-regulation gene. $(\mathrm{H}) \mathrm{q}$-RT-PCR for the top 5 down-regulation gene. * Pख0.05, **Pष0.01, ***Pष0.001. 
(A)

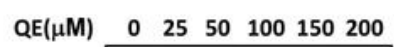

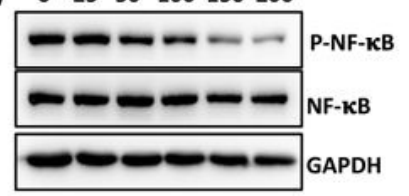

(C)

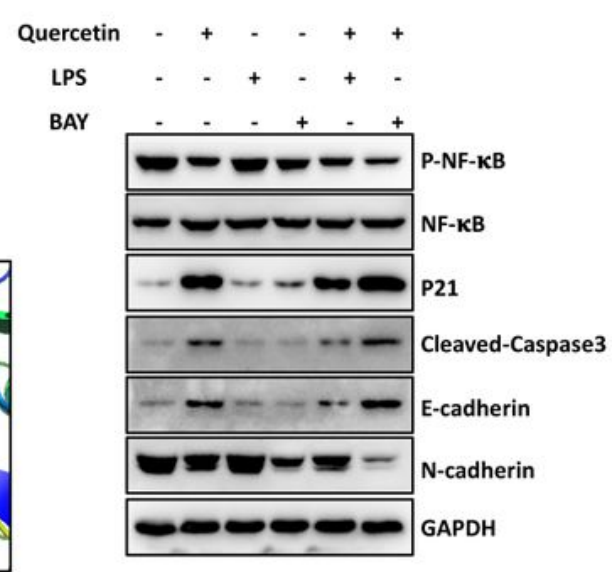

Quercetin $\mathrm{BN}=-7.6 \mathrm{kcal} / \mathrm{mol}$

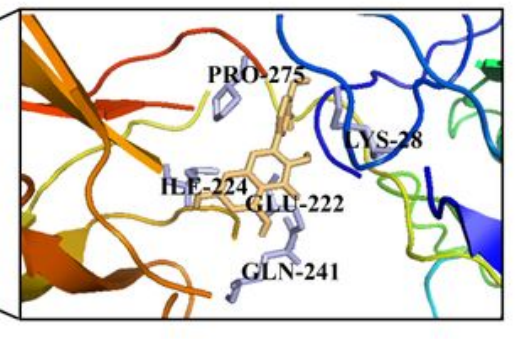

(D)
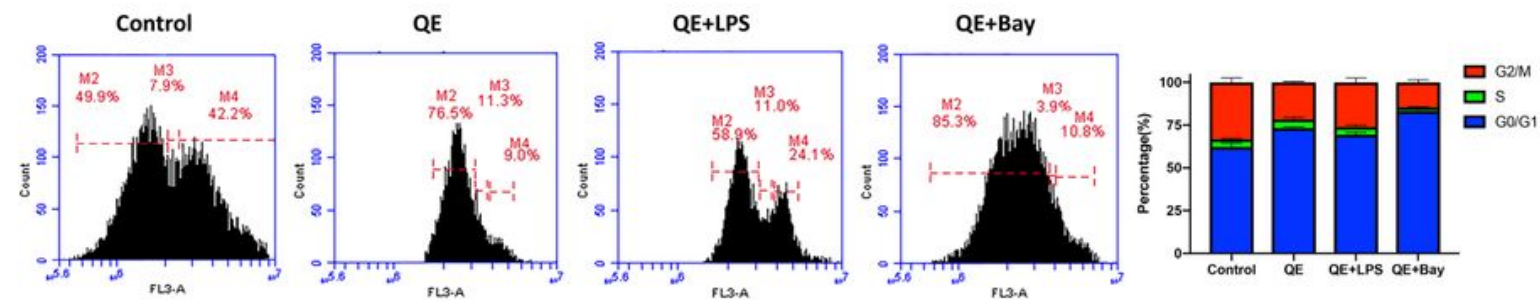

(E)
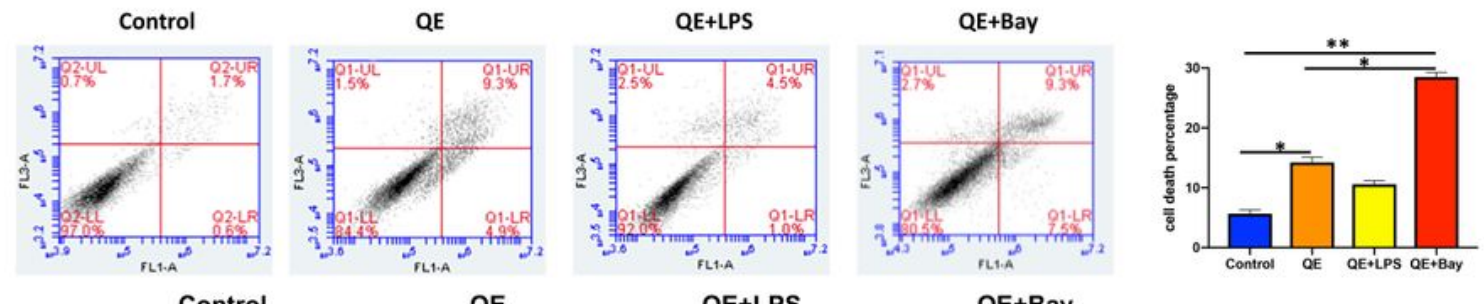

(F)
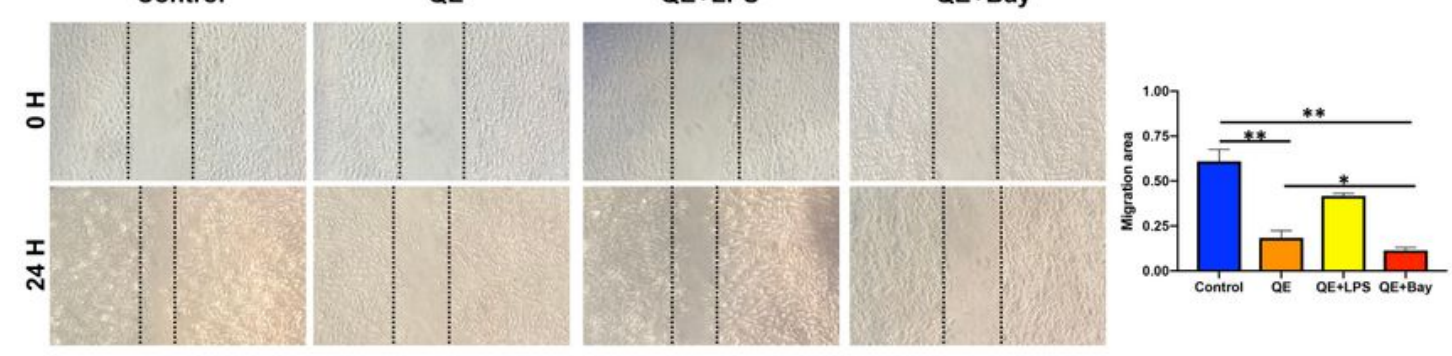

(G)

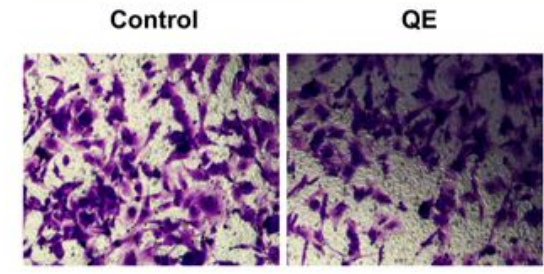

QE+LPS

QE+Bay
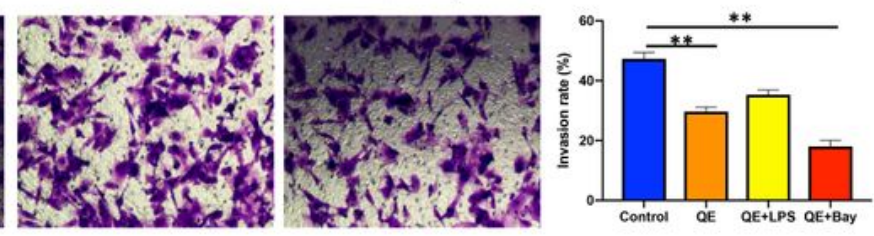

\section{Figure 5}

QE inhibited ICC by inhibiting NF- $\mathrm{B}$. (A) p-NF-KB was down-regulated by QE treatment in a dosedependent manner. (B) molecular docking with QE and NF-KB. (C) P-NF-KB, P21, Cleaved-caspase3, Ecadherin, $\mathrm{N}$ - cadherin were regulated by co-treatment with NF-KB activator (LPS) or co-treatment with NFKB inhibitor (Bay). (D) The cell cycle distribution of HuCCT1 cells co-treating with QE and LPS or Bay. (E) The cell apoptosis of HuCCT1 cells co-treating with QE and LPS or Bay. (F) The migration of HuCCT1 
cells co-treating with QE and LPS or Bay. (G) The invasion of HuCCT1 cells co-treating with QE and LPS or Bay. * Pख0.05, **Pख0.01.

(A)

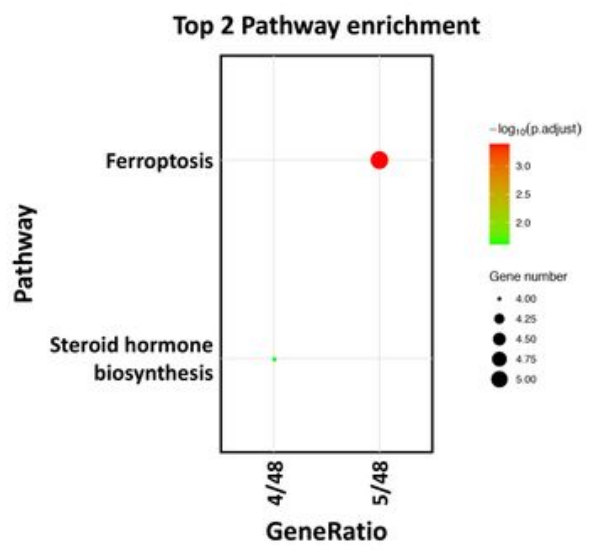

(B)

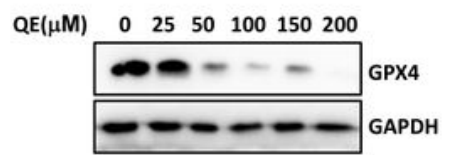

(D)

(C)
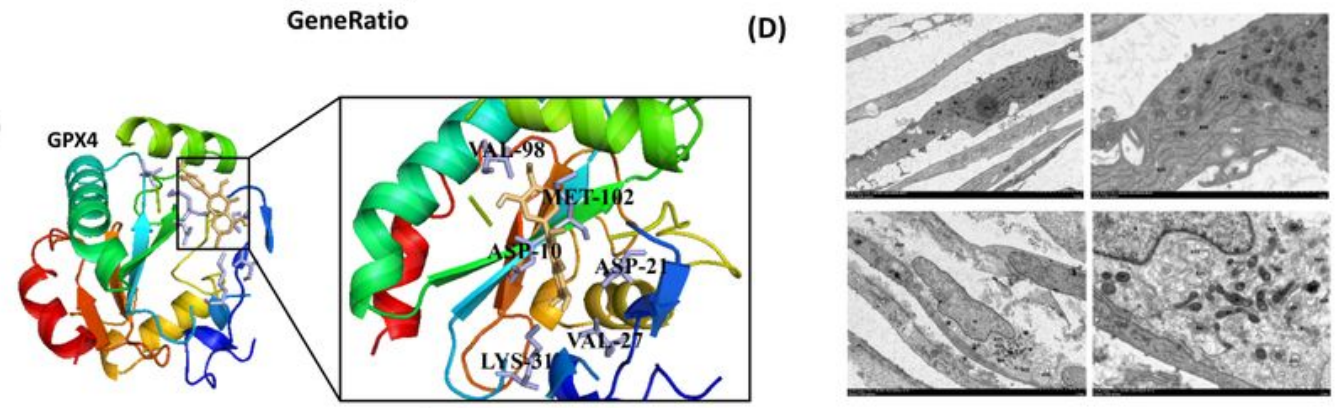

Quercetin $B N=-6.6 \mathrm{kcal} / \mathrm{mol}$

(E)

(F)

(G)
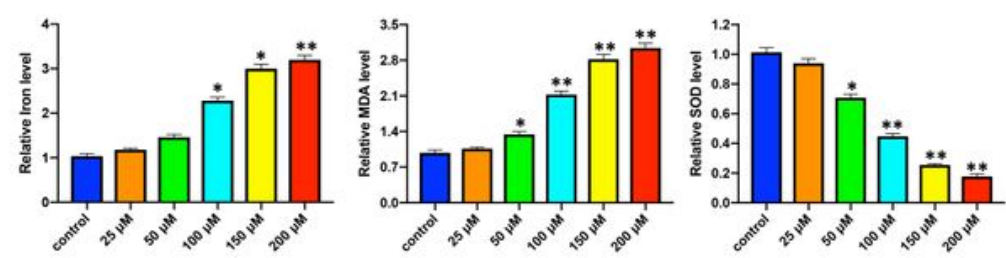

(H)

(I)

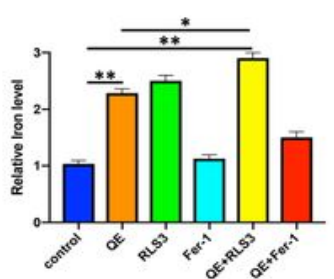

(K)

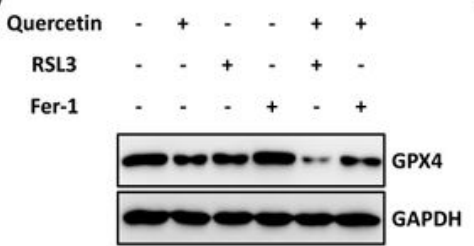

(J)

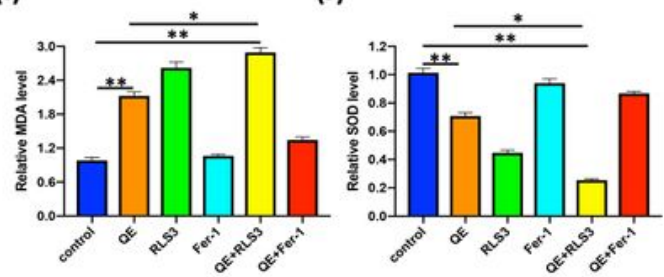

(L)

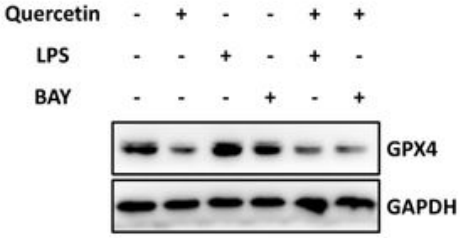

Figure 6

QE promoted ferroptosis in ICC cells. (A)RNA-seq revealed the top 2 Pathway enrichment. (B) GPX4 was down-regulated by QE treatment in a dose-dependent manner. (C) molecular docking with QE and GPX4. (D) HuCCT1 cells treated with QE showed obvious signs of ferroptosis by TME. (E) the iron level of 
HuCCT1 cells treating with QE. (F) MDA level of HuCCT1 cells treating with QE. (G) SOD level of HuCCT1 cells treating with QE. $(\mathrm{H})$ the iron level of HuCCT1 cells treating co-treating with QE and RLS3 or Fer-1. (I) MDA level of HuCCT1 cells co-treating with QE and RLS3 or Fer-1. (J) SOD level of HuCCT1 cells cotreating with QE and RLS3 or Fer-1. (K) GPX4 expression of HuCCT1 cells co-treating with QE and RLS3 or Fer-1. (L) GPX4 expression of HuCCT1 cells co-treating with QE and LPS or Bay. * Pख0.05, **Pख0.01.

(A)

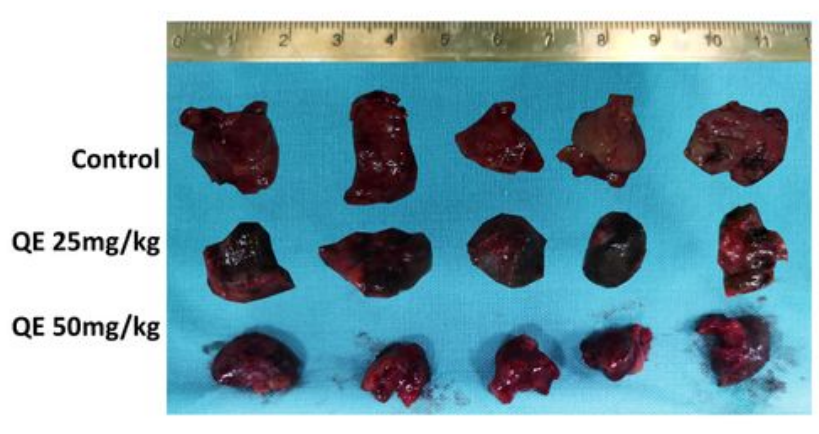

(D)

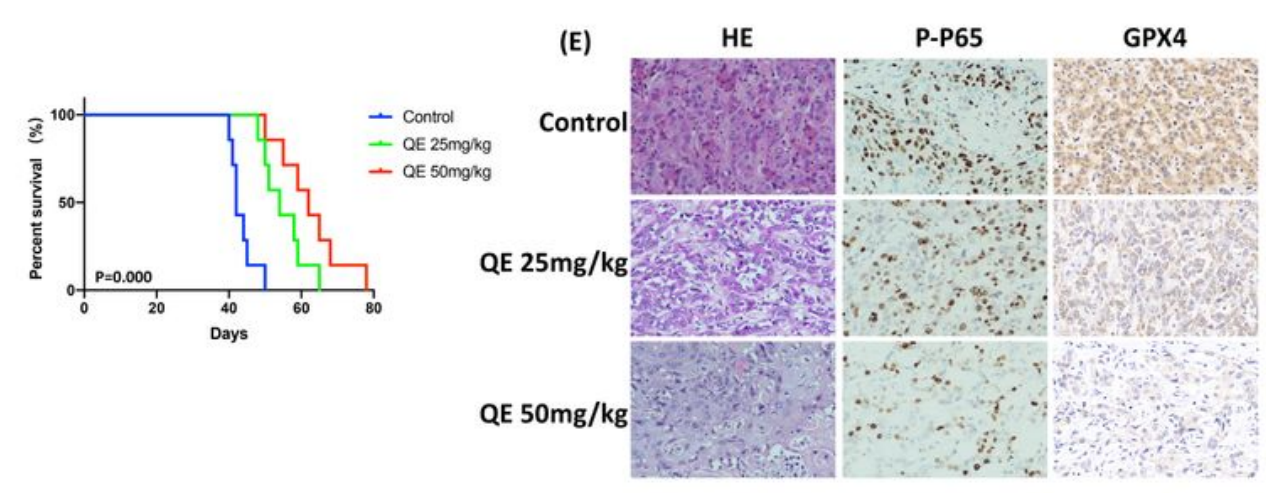

(F)

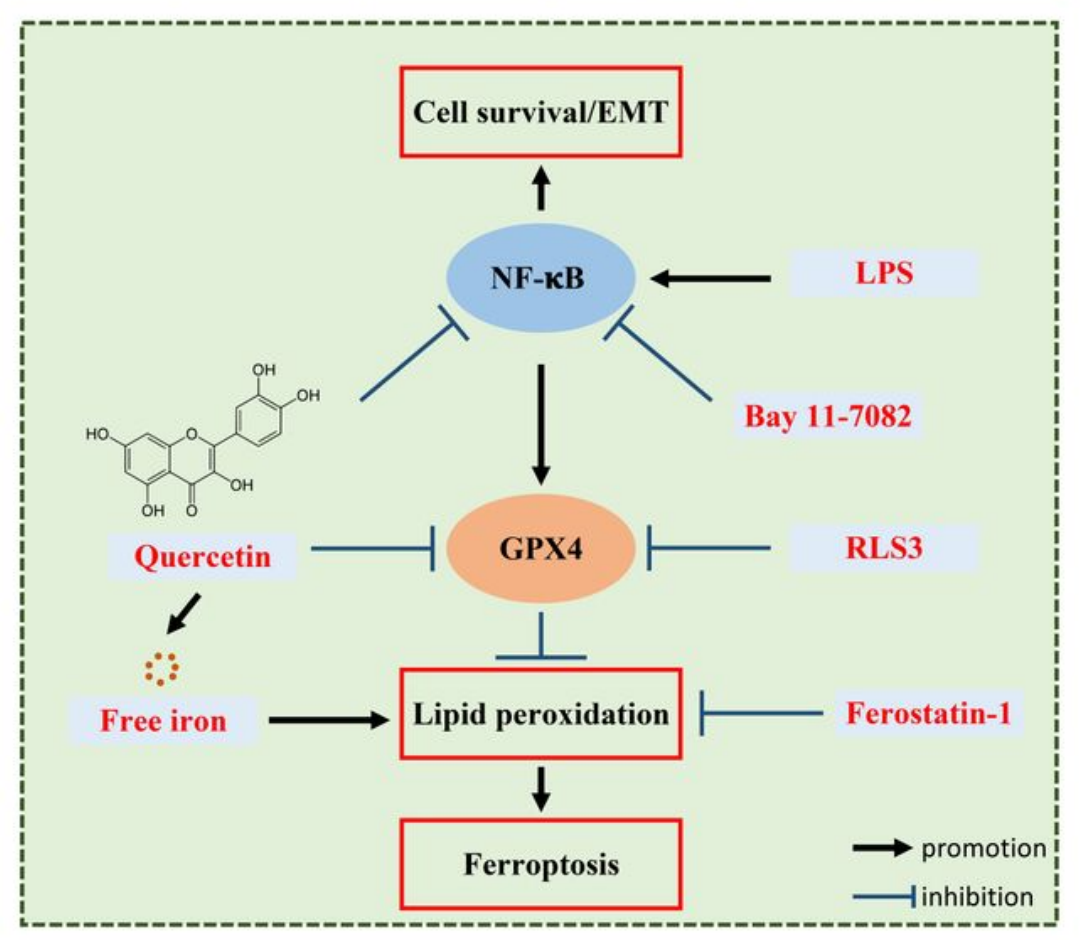

(B)
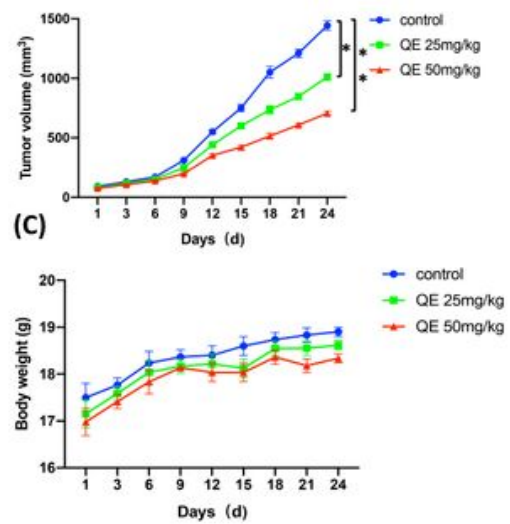

P-P65 GPX4 
Verification of the anti-tumor effect of QE in vivo. (A) the tumor size of mice with QE treatment. (B) the tumor volume of mice with QE treatment. (C) the body weight of mice with QE treatment. (D) the overall survival of mice with QE treatment. (E) HE and IHC staining with NF-DB and GPX4. (F) the summary that $\mathrm{QE}$ is a new ferroptosis inducer and combinative treatment of inhibiting NF- $\mathrm{B}$ in ICC cells by inducing ferroptosis and inhibiting EMT. * $\mathrm{P} \otimes 0.05, * * P \llbracket 0.01$.

\section{Supplementary Files}

This is a list of supplementary files associated with this preprint. Click to download.

- SupplementaryTable1PrimersforRTPCRAnalysis.docx

- SupplementaryTable2Differentiallyexpressedgenes.xIs

- SupplementaryTable3Moleculardockingdata.xls 